\title{
Erratum to: Refinement-Based OWL Class Induction with Convex Measures
}

\author{
David Ratcliffe ${ }^{1,2(\bowtie)}$ and Kerry Taylor ${ }^{1}$ \\ ${ }^{1}$ College of Engineering and Computer Science, Australian National University, \\ Canberra, ACT 2601, Australia \\ \{david.ratcliffe, kerry.taylor\}@anu.edu.au \\ 2 CSIRO Data61, GPO Box 1700, Canberra, ACT 2601, Australia
}

\section{Erratum to: \\ Chapter "Refinement-Based OWL Class Induction with Convex Measures" in: Z. Wang et al. (Eds.): Semantic Technology, LNCS 10675, https://doi.org/10.1007/978-3-319-70682-5_4}

In the original version of the paper the results reported for OWL-Miner and DL-Learner in Section 5 were incorrect, because the authors had made errors in preparing the data for the mutagenesis experiment. In the updated version of the paper, corrections were made (mainly in Section 5) to report results based on the corrected mutagenesis data. 